\title{
Structural results for planar sets with many similar subsets
}

\author{
${ }^{1}$ Bernardo M. Ábrego \\ bernardo.abrego@csun.edu \\ ${ }^{2}$ György Elekes \\ ${ }^{1}$ Silvia Fernández-Merchant \\ elekes@cs.elte.hu \\ silvia.fernandez@csun.edu \\ ${ }^{1}$ California State University, Northridge. ${ }^{2}$ Eötvös University.
}

April 2002

\begin{abstract}
Consider a $k$-element subset $P$ of the plane. It is known that the maximum number of sets similar to $P$ that can be found among $n$ points in the plane is $\Theta\left(n^{2}\right)$ if and only if the cross ratio of any quadruplet of points in $P$ is algebraic [3], [9].

In this paper we study the structure of the extremal $n$-sets $A$ which have $c n^{2}$ similar copies of $P$. As our main result we prove the existence of large lattice-like structures in such sets $A$. In particular we prove that, for $n$ large enough, $A$ must contain $m$ points in a line forming an arithmetic progression, or $m \times m$ lattices, when $P$ is not cocyclic or collinear. On the other hand we show that for cocyclic or collinear sets $P$, there are $n$-element sets $A$ with $c_{P} n^{2}$ copies of $P$ and without $k \times k$ lattice subsets.
\end{abstract}

\section{Introduction}

We identify the plane with the field of complex numbers $\mathbb{C}$. For $A, B \subseteq \mathbb{C}$ and $z, w \in \mathbb{C}$ we denote $z A=\{z a: a \in A\}$, and $A+w=\{a+w: a \in A\}$. Also, we say that $A$ and $B$ are similar, and we write $A \sim B$, if there are complex numbers $w$ and $z \neq 0$ such that $B=z A+w$.

For every pattern set $P$ and finite set $A \subset \mathbb{C}$ we define

$$
S_{P}(A)=|\{X \subseteq A: X \sim P\}| .
$$

It is a natural question, posed many times by Erdős and Purdy [4]-[6], to determine or estimate the following function

$$
S_{P}(n)=\max _{|A|=n} S_{P}(A)
$$

i.e., the maximum number of subsets similar to a given pattern $P$ that can be found among $n$ points in the plane. In [3] Erdös and the second author started the study of this function. They noticed that $S_{P}(n) \leq n(n-1)$ (a set similar to $P$ is determined by the location of any pair of reference points). They also proved that $S_{P}(n) \geq c n^{2-b \log ^{-a} n}$ for some constants $a, b, c>0$ depending only on $P$, and $S_{P}(n)=\Theta\left(n^{2}\right)$ when $P$ is an algebraic set or $|P|=3$. Later, Laczkovich and Ruzsa [9] proved that $S_{P}(n)=\Theta\left(n^{2}\right)$ if and only if the cross ratio among every quadruplet of distinct elements in $P$ is algebraic. Recall that the cross ratio of a quadruplet $(a, b, c, d)$ of different complex numbers is given by

$$
(a ; b ; c ; d)=\frac{(c-a)(d-b)}{(d-a)(c-b)} .
$$

Even though this settles the order of magnitude for a big class of sets $P$, it is not known if $\lim _{n \rightarrow \infty} S_{P}(n) / n^{2}$ exists for any non-trivial sets $P$, e.g., triangles. 
In this article we take a qualitative approach to the problem. For a pattern set $P$ satisfying $S_{P}(n)=\Theta\left(n^{2}\right)$, we say that $A$ is a $(P, c)$-rich set if $S_{P}(A) \geq c|A|^{2}$. Our objective is to find structural properties about $(P, c)$-rich sets. To accomplish this, we introduce in Section 2 the notion of a $G_{P}(m)$ set. Then we state our main result, Theorem 1, which asserts that every sufficiently large $(P, c)$-rich set must contain a $G_{P}(m)$ set. The importance of this result is seen through its corollaries. We prove, for example, the existence of large arithmetic progressions or lattice structures (when $P$ is not cocyclic or collinear) among $(P, c)$-rich sets. Other corollaries include the fact that the related function $S_{P}^{g e n}(n)$, where the maximum in (1) is restricted to sets in general position, satisfies $S_{P}^{g e n}(n)=o\left(n^{2}\right)$; and the existence of regular triangle lattices among sufficiently large extremal sets of the function $S_{T}(n)$, where $T$ is an equilateral triangle.

For the sake of continuity, we postpone the proof of Theorem 1 to Section 4, where we introduce the reader to some necessary Number Theory results by Freiman [7], Balog, Szemerédi [2], Laczkovich, Ruzsa [9], and Fürstenberg, Katznelson [8].

Finally, in Section 3, we look closely to the case when $P$ is a cocyclic or a collinear set. For this case we construct $(P, c)$-rich sets contained in at most $|P|$ lines. From this construction we characterize cocyclic or collinear sets as the only sets $P$ for which there are arbitrarily large $(P, c)$ rich sets avoiding $|P| \times|P|$-lattice structures.

\section{$2 \quad P$-generated sets and the main result}

We start this section by introducing the concept of a $G_{P}(m)$ set which will be essential for our main result. From now on $P=\left\{p_{1}, p_{2}, \ldots p_{k}\right\}$ will be the pattern set, and for every positive integer $m, I_{m}=\{j \in \mathbb{Z}:|j| \leq m\}$ and $[m]=\{j \in \mathbb{Z}: 1 \leq j \leq m\}$.

We say that $A$ is a $P$-generated set of parameter $m$, for short $G_{P}(m)$ set, if for some triplet of points in $P$, say $p_{1}, p_{2}, p_{3}$, there exist complex numbers $u, v$, and $z \neq 0$ such that $A=\bigcup_{j=1}^{k} L_{j}$ where

$$
L_{j}=\frac{p_{1}-p_{j}}{p_{1}-p_{3}}\left(u+z I_{m}\right)+\frac{p_{2}-p_{j}}{p_{2}-p_{3}}\left(v+z I_{m}\right)
$$

A $G_{P}(m)$ set has the property that whenever we 'place' a similar copy of $P$ in such a way that $p_{1} \in L_{1}$ and $p_{2} \in L_{2}$, then $p_{j} \in L_{j}$ for all $j \geq 3$ (hence the term 'generated'). We make this precise in the following proposition.

Proposition 1 For $\alpha, \beta \in I_{m}$ and $1 \leq j \leq k$ let

$$
a_{j, \alpha, \beta}=\frac{p_{1}-p_{j}}{p_{1}-p_{3}}(u+z \alpha)+\frac{p_{2}-p_{j}}{p_{2}-p_{3}}(v+z \beta) \in L_{j} .
$$

If $a_{1, \alpha, \beta} \neq a_{2, \alpha, \beta}$ the set $\left\{a_{1, \alpha, \beta}, a_{2, \alpha, \beta}, a_{3, \alpha, \beta}, \ldots, a_{k, \alpha, \beta}\right\}$ is similar to $P$.

Proof. For fixed $\alpha$ and $\beta$ we have

$$
a_{j, \alpha, \beta}=\frac{p_{1}}{p_{1}-p_{3}}(u+z \alpha)+\frac{p_{2}}{p_{2}-p_{3}}(v+z \beta)+\left(\frac{u+z \alpha}{p_{3}-p_{1}}+\frac{v+z \beta}{p_{3}-p_{2}}\right) p_{j}=u_{\alpha, \beta}+z_{\alpha, \beta} p_{j}
$$

where $u_{\alpha, \beta}$ and $z_{\alpha, \beta}$ do not depend on $j$ and $z_{\alpha, \beta} \neq 0$ since, by assumption $a_{1, \alpha, \beta} \neq a_{2, \alpha, \beta}$.

By definition $L_{1}, L_{2}$ and $L_{3}$ are arithmetic progressions of lengths $2 m+1,2 m+1$ and $4 m+1$ respectively. On the other hand, for $j \geq 4$ the set $L_{j}$ is generated by $\frac{p_{1}-p_{j}}{p_{1}-p_{3}} z$ and $\frac{p_{2}-p_{j}}{p_{2}-p_{3}} z$. It turns out that these vectors are parallel if and only if $\left\{p_{1}, p_{2}, p_{3}, p_{j}\right\}$ lie on a circle or a line. Hence 
$L_{j}$ is entirely contained in a line whenever $p_{1}, p_{2}, p_{3}$, and $p_{j}$ are cocyclic or collinear, and it is a $(2 m+1) \times(2 m+1)$ lattice otherwise.

If $P$ is a triangle and $A$ is a $G_{P}(m)$ set corresponding to the values $u=v=0$ and $z=1$, then by the above proposition we have $S_{P}(A) \geq(2 m+1)^{2}-1$. Also, by our previous remark, $n=|A|=8 m+1$. Thus $A$ is an $n$-element set with at least $n^{2} / 16$ triplets similar to $P$. This is a minor improvement over the $n^{2} / 18$ construction given in [3]; which can in fact be seen as a proper subset of $A$.

The following theorem is the main result of the paper. It describes the structure of sets in the plane with a large number of subsets similar to $P$ by showing the existence of large $G_{P}(m)$ subsets. The proof of the theorem will be postponed to Section 4. Before that, we will explore some interesting geometric consequences.

Theorem 1 For every $c>0$ and $m$ positive integer there is a threshold $N_{0}=N_{0}(c, m)$ with the following property.

Every $(P, c)$-rich set with $n>N_{0}$ elements must contain a $G_{P}(m)$ set.

Proof. See Section 4.

Corollary 1 If $S_{P}^{\prime}(n, m)$ denotes the maximum number of subsets similar to $P$, among all $n$ element subsets of the plane with no $m$ points on a line, then for every fixed $m$ we have $S_{P}^{\prime}(n, m)=$ $o\left(n^{2}\right)$.

Proof. Follows directly from Theorem 1 and the fact that any $G_{P}(m)$ set has $m$ collinear points.

As a further corollary we also have $S_{P}^{g e n}(n)=o\left(n^{2}\right)$, where this last function is the maximum number of subsets similar to $P$ among $n$-sets in general position (no 3 on a line no 4 on a circle). The next corollary states the existence of large lattices among $(P, c)$-rich sets when $P$ is not cocyclic or collinear.

Corollary 2 If $P$ is a finite set and $\left\{p_{1}, p_{2}, p_{3}, p_{4}\right\} \subseteq P$ is not a cocyclic or a collinear set then for every $c>0$ and $m$ positive integer there is a threshold $N_{0}=N_{0}(c, m)$ with the following property.

Every $(P, c)$-rich set with $n>N_{0}$ elements must contain $a(2 m+1) \times(2 m+1)$ lattice with generators $x$ and $y$ satisfying $x / y=\left(p_{1} ; p_{2} ; p_{3} ; p_{4}\right)$.

Proof. Assume without loss of generality that $p_{1}=0$ and $p_{2}=1$. Suppose $Q$ is a $(P, c)$-rich set with $|Q|=n>N_{0}$, where $N_{0}$ is given by Theorem 1 . Hence $Q$ must contain a $G_{P}(m)$ set, so in particular $Q$ contains the set

$$
\begin{aligned}
L_{4} & =\frac{p_{4}}{p_{3}}\left(u+z I_{m}\right)+\frac{1-p_{4}}{1-p_{3}}\left(v+z I_{m}\right) \\
& =\left(\frac{p_{4}}{p_{3}} u+\frac{1-p_{4}}{1-p_{3}} v\right)+\frac{p_{4}}{p_{3}} z I_{m}+\frac{1-p_{4}}{1-p_{3}} z I_{m} .
\end{aligned}
$$

Let $x=\frac{1-p_{4}}{1-p_{3}} z, y=\frac{p_{4}}{p_{3}} z$. Notice that $x / y=\left(0 ; 1 ; p_{3} ; p_{4}\right)$ and since $\left\{p_{1}, p_{2}, p_{3}, p_{4}\right\}$ is not a cocyclic set then $\left(0 ; 1 ; p_{3} ; p_{4}\right) \notin \mathbb{R}$, and consequently $x$ and $y$ are linearly independent. Thus $L_{4}$ is the required lattice.

There are $n$-sets with $\mathrm{cn^{2 }}$ triples determining equilateral triangles, and containing no $3 \times 3$ lattices (e.g. any $G_{P}(m)$ set with $n$ points and $P$ an equilateral triangle). But large lattices are unavoidable whenever $c>1 / 6$, more precisely we have the following. 
Corollary 3 Let $T$ be an equilateral triangle. For $m$ positive integer and $\varepsilon>0$ there is a threshold $N_{1}=N_{1}(\varepsilon, m)$ with the following property.

Every set $A$ with $n>N_{1}$ elements for which $S_{T}(A) \geq\left(\frac{1}{6}+\varepsilon\right) n^{2}$ must contain a $(2 m+1) \times$ $(2 m+1)$ regular triangle lattice.

Proof. Let $T$ be the triangle $\left\{0,1, e^{i \pi / 3}\right\}$ and $R$ the rhomb $\left\{0,1, e^{i \pi / 3}, e^{-i \pi / 3}\right\}$. Suppose $A$ is an $n$-element set satisfying $S_{T}(A) \geq\left(\frac{1}{6}+\varepsilon\right) n^{2}$. For $i=1,2$ define $X_{i}$ as the number of pairs in $A$ that are the vertices of exactly $i$ equilateral triangles with vertices in $A$. Observe that according to our definitions $S_{T}(A)=\frac{1}{3} X_{1}+\frac{2}{3} X_{2}$ and $S_{R}(A)=X_{2}$, hence

$$
X_{1}+2 X_{2} \geq\left(\frac{1}{2}+3 \varepsilon\right) n^{2}
$$

and using the trivial inequality $X_{1}+X_{2}<(1 / 2) n^{2}$ we conclude that

$$
S_{R}(A)=X_{2}>3 \varepsilon n^{2} .
$$

Hence by Corollary 2, for $n>N_{1}=N_{0}(3 \varepsilon, m)$ we have that $A$ contains a $(2 m+1) \times(2 m+1)$ lattice with generators $x$ and $y$ satisfying $x / y=\left(0 ; 1 ; e^{i \pi / 3} ; e^{-i \pi / 3}\right)=-e^{i \pi / 3}$, so the lattice is in fact a regular triangle lattice.

As a further corollary we can remark that in [1] it was proved that $S_{T}(n) \geq\left(\frac{1}{3}-\frac{\sqrt{3}}{4 \pi}\right) n^{2}+$ $O\left(n^{3 / 2}\right)$, so the extremal sets for the function $S_{T}(n)$ contain large regular lattices.

When the set $P$ is cocyclic Theorem 1 cannot guarantee a lattice substructure, but in that case we can always obtain a set of $k$ concurrent lines with many points.

Corollary $4 A$ set of arithmetic progressions in $\mathbb{C}$ is said to be concurrent if the lines containing the progressions are concurrent. If $P$ is a cocyclic set with $k$ elements then for every $c>0$ and $m$ positive integer there is a threshold $N_{0}=N_{0}(c, m)$ with the following property.

Every $(P, c)$-rich set with $n>N_{0}$ elements must contain a set of $k$ concurrent arithmetic progressions each of size $2 m+1$.

Proof. Let $g_{j}=\left(p_{1} ; p_{2} ; p_{3} ; p_{j}\right)$ for $j \geq 2$. Assume again without loss of generality that $p_{1}=0$ and $p_{2}=1$. Since $P$ is a cocyclic set then we know that $g_{j} \in \mathbb{R}$ for all $j \geq 3$. Suppose $Q$ is a $(P, c)$-rich set with $|Q|=n>N_{0}$, where $N_{0}$ is given by Theorem 1 . Thus $Q$ contains a $G_{P}(m)$ set. Define the lines

$$
\begin{aligned}
\ell_{1} & =\frac{v}{1-p_{3}}+\frac{z}{1-p_{3}} \mathbb{R} \text { and } \\
\ell_{j} & =\left(u+g_{j} v\right)+\frac{z p_{j}}{p_{3}} \mathbb{R} \text { for } j \geq 2 .
\end{aligned}
$$

Clearly $L_{j} \subseteq \ell_{j}$ and every $L_{j}$ contains a $2 m+1$ arithmetic progression. Now consider the similarity transformation $T: \mathbb{C} \rightarrow \mathbb{C}$ given by $T(w)=\frac{p_{3}}{z} w-\frac{u}{z}$. By definition we have

$$
\begin{aligned}
& T\left(\ell_{1}\right)=x+\frac{p_{3}}{1-p_{3}} \mathbb{R} \text { and } \\
& T\left(\ell_{j}\right)=x\left(1-p_{j}\right)+p_{j} \mathbb{R} \text { for } j \geq 2,
\end{aligned}
$$


where $x=\frac{p_{3}(u+v)-u}{z\left(1-p_{3}\right)}$. A simple calculation shows that $R_{j}:=T\left(\ell_{j}\right) \cap T\left(\ell_{2}\right)$ is given by

$$
\begin{aligned}
R_{1} & =\frac{p_{3}\left(1-\overline{p_{3}}\right) \bar{x}-\overline{p_{3}}\left(1-p_{3}\right) x}{p_{3}-\overline{p_{3}}} \text { and } \\
R_{j} & =\frac{p_{j}\left(\bar{x}+x \overline{p_{j}}\right)-\overline{p_{j}}\left(x+\bar{x} p_{j}\right)}{p_{j}-\overline{p_{j}}} \text { for } j \geq 3 .
\end{aligned}
$$

Using the fact that $g_{j} \in \mathbb{R}$ and $p_{j}=p_{3}\left(p_{3}+g_{j}\left(1-p_{3}\right)\right)^{-1}$ we obtain for $j \geq 3$

$$
R_{j}=\frac{p_{3}\left(1-\overline{p_{3}}\right) \bar{x}-\overline{p_{3}}\left(1-p_{3}\right) x}{p_{3}-\overline{p_{3}}} .
$$

Therefore the set of lines $\left\{\ell_{1}, \ell_{2}, \ldots, \ell_{k}\right\}$ is concurrent.

\section{Cocyclic and collinear patterns}

To complement the results from last section we present the following theorem, where for $P$ a cocyclic set, we construct a set contained in $k$ concurrent lines and with many similar copies of $P$.

The proof of the theorem mimics, in a certain sense, the construction first done in [3]. The main difference being the use of the cross ratios $\left(p_{1} ; p_{2} ; p_{3} ; p_{j}\right)$ instead of the value $p_{j}$, this allows our set to be contained in a set of at most $k$ lines as opposed to have the grid-like structure described in [3].

Theorem 2 For every $k$-element cocyclic or collinear set $P$ with $k \geq 3$ and $S_{P}(n)=\Theta\left(n^{2}\right)$, there is a constant $c=c(P)>0$ and a collection of lines $\ell_{1}, \ell_{2}, \ldots, \ell_{k}$ through the origin satisfying the following property.

For every integer $n \geq k$ there is a n-element set $A_{n} \subset \bigcup_{j=1}^{k} \ell_{j}$ satisfying $S_{P}\left(A_{n}\right) \geq c n^{2}$.

Before we see the proof of the theorem let us state the following consequence which characterizes the notion of cocyclicity (or collinearity) for finite sets.

Corollary 5 For every k-element set $P$ satisfying $S_{P}(n)=\Theta\left(n^{2}\right)$ the following are equivalent.

1. $P$ is cocyclic or collinear.

2. There is a constant $c>0$ and arbitrarily large $(P, c)$-rich sets containing no $k \times k$ lattices.

Proof. Trivial for $k \leq 2$. The implication $(1) \Rightarrow(2)$ follows from Theorem 2 and the fact that a set contained in $k$ concurrent lines cannot contain a $k \times k$ lattice. (2) $\Rightarrow(1)$ follows directly from Corollary 2 using any value $m \geq(k-1) / 2$.

Proof of Theorem 2. Assume without loss of generality that $p_{1}=0$ and $p_{2}=1$. Let $g_{2}=0$, $g_{3}=1$, and $g_{j}=\left(0 ; 1 ; p_{3} ; p_{j}\right)$ for $4 \leq j \leq k$; note that $g_{j} \in \mathbb{R}$ since $P$ is cocyclic or collinear. Since $S_{P}(n)=\Theta\left(n^{2}\right)$, we have that by Laczkovich-Ruzsa Characterization Theorem [9] every $g_{j}$ is algebraic. Let $D$ be the degree of $L=\mathbb{Q}\left(g_{3}, g_{4}, \ldots, g_{k}\right)$ over $\mathbb{Q}$ and $\left\{h_{1}, h_{2}, \ldots, h_{D}\right\}$ be a basis of $L$ over $\mathbb{Q}$. 
We first prove the theorem for an increasing sequence $\left\{n_{m}\right\}$ of values of $n$. For every $m \geq 1$ consider the following set of real numbers

$$
\mathcal{G}_{m}=\left\{\sum_{i=1}^{D} a_{i} h_{i}: a_{i} \in I_{m}\right\} .
$$

Clearly $\left|\mathcal{G}_{m}\right|=(2 m+1)^{D}$. Let $J_{m}=\mathcal{G}_{m} \times \mathcal{G}_{m} \backslash\left\{(a, b) \in \mathcal{G}_{m} \times \mathcal{G}_{m}: b p_{3}-a=0\right\}$. For every $(a, b) \in$ $J_{m}$ define the similarity transformation

$$
T_{a, b}(z)=\frac{p_{3}(a-b)}{p_{3}-1}+\frac{b p_{3}-a}{p_{3}-1} z .
$$

Let $n_{m}=\left|\bigcup_{\substack{(a, b) \in J_{m} \\ 1 \leq j \leq k}} T_{a, b}\left(p_{j}\right)\right|$ and $A_{n_{m}}=\bigcup_{\substack{(a, b) \in J_{m} \\ 1 \leq j \leq k}} T_{a, b}\left(p_{j}\right)$. By definition we have

$$
T_{a, b}(0)=\frac{p_{3}}{p_{3}-1}(a-b), \text { and } T_{a, b}\left(p_{j}\right)=p_{j}\left(a\left(1-g_{j}\right)+b g_{j}\right) \text { for } j \geq 2 .
$$

Let $\ell_{1}, \ell_{2}, \ldots, \ell_{k}$ be a collection of lines defined as follows,
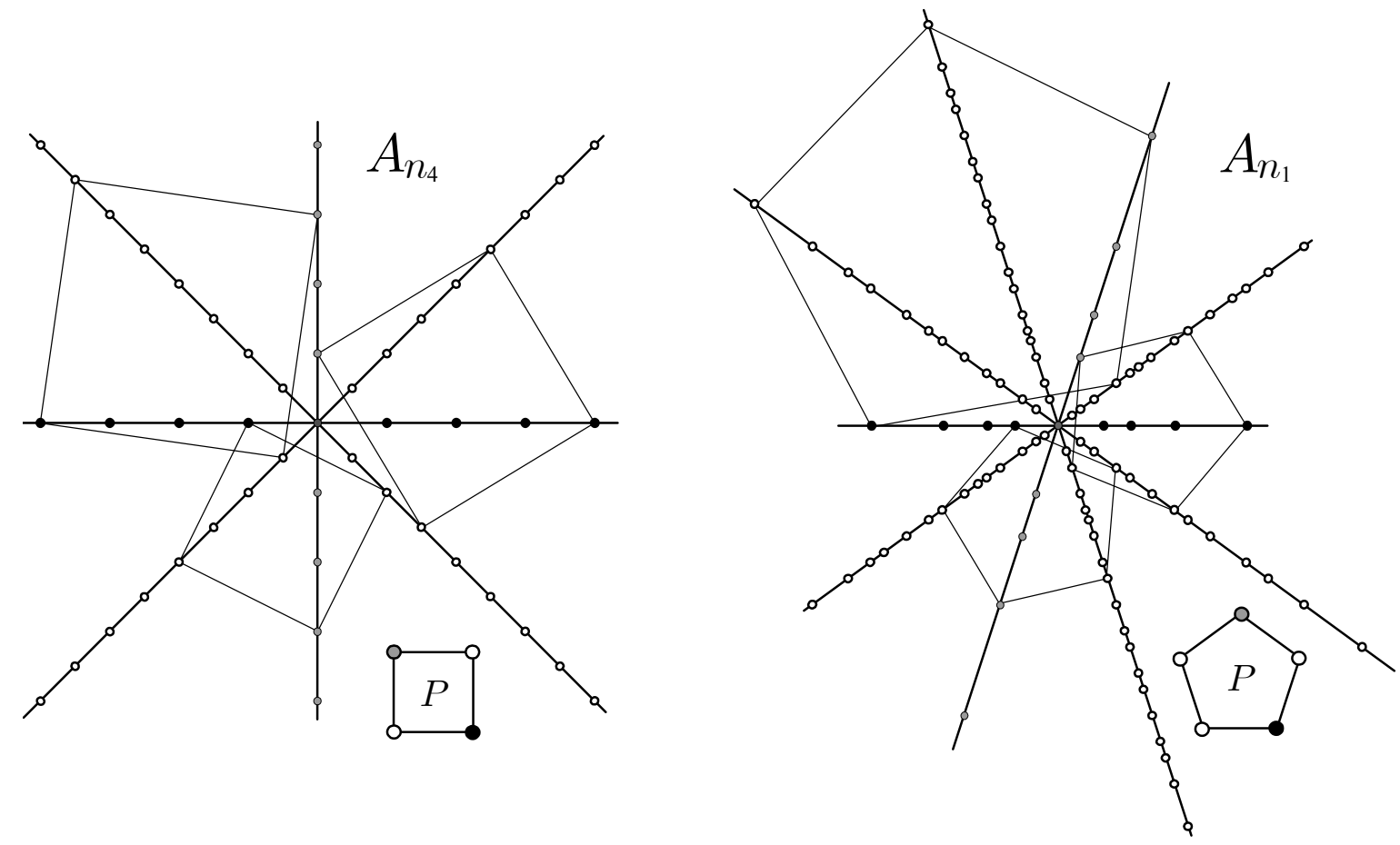

Figure 1: Examples of the sets $A_{n_{m}}$ for some sets $P$.

$$
\ell_{1}:=\left(\frac{p_{3}}{p_{3}-1}\right) \mathbb{R}, \text { and } \ell_{j}:=p_{j} \mathbb{R} \text { for } j \geq 2
$$

By construction we have that for every $1 \leq j \leq k$

$$
\bigcup_{(a, b) \in J_{m}} T_{a, b}\left(p_{j}\right) \subseteq \ell_{j}
$$


thus $A_{n_{m}} \subset \bigcup_{j=1}^{k} \ell_{j}$ and $T_{a, b}(P) \subset A_{n_{m}}$ for every $(a, b) \in J_{m}$.

Now, if $P$ is cocyclic then all the lines in (3) are pairwise different which together with (2) gives $T_{a, b}(P) \neq T_{a^{\prime}, b^{\prime}}(P)$ whenever $(a, b) \neq\left(a^{\prime}, b^{\prime}\right)$. On the other hand, if $P$ is collinear we can assume $p_{1}=0<p_{3}<p_{4}<\ldots<p_{k}<1=p_{2}$. If $T_{a, b}(P)=T_{a^{\prime}, b^{\prime}}(P)$ then either $(a, b)=\left(a^{\prime}, b^{\prime}\right)$ or by $(2)$ $P$ is symmetric with respect to $1 / 2$ and $T_{a, b}^{-1} \circ T_{a^{\prime}, b^{\prime}}(z)$ is a half-turn rotation with center $1 / 2$. In both cases, for each pair $(a, b)$ there is at most one pair $\left(a^{\prime}, b^{\prime}\right) \neq(a, b)$ such that $T_{a, b}(P)=T_{a^{\prime}, b^{\prime}}(P)$ which means

$$
S_{P}\left(A_{n_{m}}\right) \geq \frac{1}{2}\left|J_{m}\right| \geq \frac{1}{2}\left(\left|\mathcal{G}_{m}\right|^{2}-\left|\mathcal{G}_{m}\right|\right) \geq \frac{1}{3}\left|\mathcal{G}_{m}\right|^{2} .
$$

Now we will find appropriate bounds for the size of $A_{n_{m}}$ in terms of $\left|\mathcal{G}_{m}\right|$. To accomplish this we need the following lemma.

Lemma 1 There are positive integers $M, K$ independent of $m$ so that for every $2 \leq j \leq k$ we have that $\left(K g_{j}\right) \mathcal{G}_{m} \subseteq \mathcal{G}_{M m}$.

Proof. Let $y \in L=\mathbb{Q}\left(g_{3}, g_{4}, \ldots, g_{k}\right)$. Express $y h_{i}$ as a linear combination of $\left\{h_{1}, h_{2}, \ldots, h_{D}\right\}$ over $\mathbb{Q}$,

$$
y h_{i}=\sum_{j=1}^{D} q_{i, j} h_{j}
$$

Suppose $q_{i, j}=a_{i, j} / b_{i, j}$ with $a_{i, j}, b_{i, j}$ integers. Let $K$ be the least common multiple of $\left\{b_{i, j}\right\}$ and $M_{0}$ the maximum of $\left\{\left|a_{i, j}\right|\right\}$. Then for every $z=\sum_{i=1}^{D} z_{i} h_{i} \in \mathcal{G}_{m}$ we have

$$
K y z=\sum_{i=1}^{D} z_{i} K y h_{i}=\sum_{j=1}^{D}\left(\sum_{i=1}^{D} \frac{z_{i} K a_{i, j}}{b_{i, j}}\right) h_{j} \in \mathbb{Z}\left(g_{3}, g_{4}, \ldots, g_{k}\right)
$$

and

$$
\left|\sum_{i=1}^{D} \frac{z_{i} K a_{i, j}}{b_{i, j}}\right| \leq \sum_{i=1}^{D}\left|\frac{z_{i} K a_{i, j}}{b_{i, j}}\right| \leq K M_{0} D m .
$$

So by letting $M=K M_{0} D$ we infer that $(K y) \mathcal{G}_{m} \subseteq \mathcal{G}_{M m}$. Clearly we can find common values of $K$ and $M$ to make this work simultaneously for $y=g_{2}, g_{3}, \ldots, g_{k}$.

Besides this lemma we also need the following easy facts for generalized arithmetic progressions of the form $\mathcal{G}_{m}$ : For any positive integers $N, m_{1}, m_{2}, m$ we have that $N \mathcal{G}_{m} \subseteq \mathcal{G}_{N m}$ and $\mathcal{G}_{m_{1}} \pm \mathcal{G}_{m_{2}}=$ $\mathcal{G}_{m_{1}+m_{2}}$. Using these properties we have

$$
\left.\left|\bigcup_{(a, b) \in J_{m}} T_{a, b}\left(p_{1}\right)\right|=\left|\mathcal{G}_{m}-\mathcal{G}_{m}\right|=\left|\mathcal{G}_{2 m}\right| \quad \text { (note that }\left|\mathcal{G}_{m}\right| \leq\left|\mathcal{G}_{2 m}\right| \leq 2^{D}\left|\mathcal{G}_{m}\right|\right)
$$

and for $j \geq 2$,

$$
\begin{aligned}
& \left|\bigcup_{(a, b) \in J_{m}} T_{a, b}\left(p_{j}\right)\right|=\left|\left(1-g_{j}\right) \mathcal{G}_{m}+g_{j} \mathcal{G}_{m}\right| \leq\left|K \mathcal{G}_{m}-K g_{j} \mathcal{G}_{m}+K g_{j} \mathcal{G}_{m}\right| \\
& \leq\left|\mathcal{G}_{K m}-\mathcal{G}_{M m}+\mathcal{G}_{M m}\right|=\left|\mathcal{G}_{(K+2 M) m}\right| \leq(K+2 M)^{D}\left|\mathcal{G}_{m}\right| \\
& \left|\bigcup_{(a, b) \in J_{m}} T_{a, b}\left(p_{j}\right)\right|=\left|\left(1-g_{j}\right) \mathcal{G}_{m}+g_{j} \mathcal{G}_{m}\right| \geq\left|\mathcal{G}_{m}\right| \text {. }
\end{aligned}
$$


So by letting $c_{1}=k$ and $c_{2}=k(K+2 M)^{D}$ we have

$$
c_{1}\left|\mathcal{G}_{m}\right| \leq n_{m} \leq c_{2}\left|\mathcal{G}_{m}\right|
$$

Hence by (4) we conclude that $S_{P}\left(A_{n_{m}}\right) \geq \frac{1}{3}\left|\mathcal{G}_{m}\right|^{2} \geq \frac{1}{3} c_{2}^{-2} n_{m}^{2}$.

To prove the result for arbitrary $n \geq k$ we note that, using (5) and $\left|\mathcal{G}_{m}\right|=(2 m+1)^{D}$, there is a constant $c_{3}$ independent of $m$ such that $n_{m}<n_{m+1} \leq c_{3} n_{m}$. Thus, if $n_{m}<n \leq n_{m+1}$ we let $A_{n}$ be the set $A_{n_{m}}$ constructed before together with $n-n_{m}$ extra points in the line $\ell_{1}$. It is clear that $S_{P}\left(A_{n}\right) \geq c_{4} n^{2}$ which completes the proof.

Remark. If $P$ is collinear all the lines in (3) are the same. If $P$ is cocyclic then all the lines in (3) are different and every similar copy of $P$ lies on a circle passing through the origin.

\section{Proof of Theorem 1}

The proof of our main result will be based on some deep results of Combinatorial Number Theory, namely those which describe the structure of small sumsets. These results are usually stated for subsets of the integers, but they are in fact true for subsets of any torsion-free abelian group [10], in particular for subsets of $\mathbb{C}$. The notion of generalized arithmetic progressions (first introduced by Szemerédi [12] in his famous paper) is involved in all of these results. For any $d$ positive integer, $\left\{n_{1}, n_{2}, \ldots, n_{d}\right\} \subseteq \mathbb{N}$, and $\left\{z_{1}, z_{2}, \ldots, z_{d}\right\} \subseteq \mathbb{C} \backslash\{0\}$, we call the set

$$
\mathcal{G}=\left\{\sum_{i=1}^{d} k_{i} z_{i}: 0 \leq k_{i}<n_{i}\right\}
$$

a generalized arithmetic progression of dimension $d$ and parameters $\left\{n_{i}\right\}$ and $\left\{z_{i}\right\}$. In what follows the symbol $\mathcal{G}_{d, n}$ will denote a generalized arithmetic progression of dimension not exceeding $d$ and size $n$. For short we will use expressions like "there exists a $\mathcal{G}_{d, n}$ ".

From now on we assume $A, B \subseteq \mathbb{C},|A|=|B|=n$, and $E \subseteq A \times B$. We write $A+B=\{a+b$ : $a \in A, b \in B\}$ and $A+{ }_{E} B=\{a+b:(a, b) \in E\}$.

Using exponential sums and methods from the geometry of numbers. Freiman obtained the following result that describes the structure of $A+B$ when the cardinality of such set is not much greater than $n$. Later Ruzsa found a simpler proof [11].

Theorem A (Freiman [7]) For every $C>0$ there is a positive constant $c=c(C)$ and a natural number $d=d(C)$ satisfying the following property.

If $|A+B| \leq C n$ then $A \cup B$ is contained in a $\mathcal{G}_{d, c n}$.

Balog and Szemerédi found a statistical version of this theorem, by relaxing the assumption that all pairwise sums must be taken into account, and just considering the sums of $\mathrm{cn}^{2}$ pairs.

Theorem B (Balog, Szemerédi [2]) For every $C_{1}, C_{2}>0$ there are positive constants $c_{1}=c_{1}\left(C_{1}, C_{2}\right)$, $c_{2}=c_{2}\left(C_{1}, C_{2}\right)$, and a natural number $d=d\left(C_{1}, C_{2}\right)$ satisfying the following property.

If $\left|A+{ }_{E} B\right| \leq C_{1} n$ and $|E| \geq C_{2} n^{2}$ then there is a $\mathcal{G}_{d, c_{1} n}$ satisfying $\left|A \cap \mathcal{G}_{d, c_{1} n}\right| \geq c_{2} n$ and $\left|B \cap \mathcal{G}_{d, c_{1} n}\right| \geq c_{2} n$.

Laczkovich and Ruzsa took this theorem a step further by proving the following generalization, which is precisely the result we use for the proof of Theorem 1. 
Theorem C (Laczkovich, Ruzsa [9]) For every $C_{1}, C_{2}>0$ there are positive constants $c_{1}=$ $c_{1}\left(C_{1}, C_{2}\right), c_{2}=c_{2}\left(C_{1}, C_{2}\right)$, and a natural number $d=d\left(C_{1}, C_{2}\right)$ satisfying the following property.

If $\left|A+{ }_{E} B\right| \leq C_{1} n$ and $|E| \geq C_{2} n^{2}$ then there is a $\mathcal{G}_{d, c_{1} n}$ satisfying

$$
\left|E \cap\left(\mathcal{G}_{d, c_{1} n} \times \mathcal{G}_{d, c_{1} n}\right)\right| \geq c_{2} n^{2} .
$$

The following lemma, which is a consequence of Theorem $\mathrm{C}$, is used in the proof of Theorem 1 . Any proof with explicit bounds of this lemma would in turn provide explicit bounds for the results in this paper.

Lemma 2 For every $C_{1}, C_{2}>0$ there is a positive constant $c_{3}=c_{3}\left(C_{1}, C_{2}\right)$ such that for every positive integer $M$, there is a threshold function $N=N\left(C_{1}, C_{2}, M\right)$ satisfying the following property.

For every $n \geq N$, if $\left|A+{ }_{E} B\right| \leq C_{1} n$ and $|E| \geq C_{2} n^{2}$ then there are arithmetic progressions $\mathcal{G}_{1}, \mathcal{G}_{2}$ of length $l \geq M$ and common difference such that

$$
\left|E \cap\left(\mathcal{G}_{1} \times \mathcal{G}_{2}\right)\right| \geq c_{3} l^{2} .
$$

Proof. By Theorem $\mathrm{C}$, applied to the sets $A$ and $B$, there are positive constants $c_{1}, c_{2}$, a positive integer $d$ (all depending on $C_{1}$ and $C_{2}$ only), and $\mathcal{G}=\mathcal{G}_{d, c_{1} n}$ a generalized arithmetic progression satisfying $|(\mathcal{G} \times \mathcal{G}) \cap E| \geq c_{2} n^{2}$. Suppose $\mathcal{G}$ is given by

$$
\mathcal{G}=\left\{\sum_{i=1}^{d} k_{i} z_{i}: 0 \leq k_{i}<n_{i}\right\}
$$

with $n_{1} \geq n_{2} \geq \ldots \geq n_{d} \geq 1$ and $\prod_{i=1}^{d} n_{i}=c_{1} n$. Let $c_{3}=c_{2} / c_{1}^{2}, N=M^{d} / c_{1}$, and $l=n_{1}$.

For every $r=\left(r_{2}, r_{3}, \ldots, r_{d}\right) \in\left[n_{2}\right] \times\left[n_{3}\right] \times \cdots \times\left[n_{d}\right]$ define

$$
\mathcal{G}_{r}=\left\{k_{1} z_{1}+\sum_{i=2}^{d} r_{i} z_{i}: 0 \leq k_{1}<n_{1}=l\right\} .
$$

Observe that

$$
\sum_{r, s \in\left[n_{2}\right] \times\left[n_{3}\right] \times \cdots \times\left[n_{d}\right]}\left|\left(\mathcal{G}_{r} \times \mathcal{G}_{s}\right) \cap E\right| \geq|(\mathcal{G} \times \mathcal{G}) \cap E| \geq c_{2} n^{2}
$$

so by an averaging argument there are $R_{1}, R_{2} \in\left[n_{2}\right] \times\left[n_{3}\right] \times \cdots \times\left[n_{d}\right]$ satisfying

$$
\left|E \cap\left(\mathcal{G}_{R_{1}} \times \mathcal{G}_{R_{2}}\right)\right| \geq\left(c_{2} / c_{1}^{2}\right) n_{1}^{2}=c_{3} l^{2} .
$$

Notice that $\mathcal{G}_{R_{1}}$ and $\mathcal{G}_{R_{2}}$ are arithmetic progressions with common difference $z_{1}$. If $n \geq N$ then $l^{d}=n_{1}^{d} \geq c_{1} n \geq c_{1} N=M^{d}$, i.e., $l \geq M$ and thus $\mathcal{G}_{R_{1}}, \mathcal{G}_{R_{2}}$ is the required pair of arithmetic progressions.

The other main ingredient for our proof is a two dimensional generalization of the famous Szemerédi Theorem [12] regarding the existence of long arithmetic progressions among subsets of $\mathbb{Z}$ with positive density. The theorem is due to Fürstenberg and Katznelson and was obtained by means of Ergodic Theory [8]. No quantitative proof of this result is known.

Theorem D (Fürstenberg, Katznelson [8]) For every integer $m$ and constant $c>0$ there is a threshold function $N_{1}=N_{1}(c, m)$ with the following property.

For every $n \geq N_{1}$, if $A \subseteq[n] \times[n]$ and $|A| \geq c n^{2}$ then $A$ contains an $m \times m$ square sublattice, i.e., a set of the form $\left(a_{1}+b[m]\right) \times\left(a_{2}+b[m]\right)$ with $a_{1}, a_{2}, b$ positive integers. 
Finally we are in a position to prove the main theorem.

Proof of Theorem 1. First assume without loss of generality that $p_{1}=0$ and $p_{2}=1$. Suppose $Q$ is a $(P, c)$-rich set of size $n$. Let $A=p_{3} Q, B=\left(1-p_{3}\right) Q$, and

$$
E=\left\{(a, b) \in A \times B: b p_{3} \neq a\left(1-p_{3}\right) \text { and } a \frac{p_{j}}{p_{3}}+b \frac{1-p_{j}}{1-p_{3}} \in Q \text { for all } j\right\}
$$

Consider the function $f: E \rightarrow\{X \subset Q: X \sim P\}$ given by

$$
f(a, b)=\left\{a \frac{p_{j}}{p_{3}}+b \frac{1-p_{j}}{1-p_{3}}: 1 \leq j \leq k\right\}=\left\{\frac{b}{1-p_{3}}+\left(\frac{a}{p_{3}}-\frac{b}{1-p_{3}}\right) p_{j}: 1 \leq j \leq k\right\} .
$$

Observe that $f$ is well defined since $a / p_{3}-b /\left(1-p_{3}\right) \neq 0$ by assumption (i.e., $\left.b p_{3} \neq a\left(1-p_{3}\right)\right)$. Let $X \subseteq Q$ satisfy $X \sim P$, thus $X=w+z P$ for some $w, z \in \mathbb{C}$ and $z \neq 0$. Let $a=p_{3}(w+z)$ and $b=w\left(1-p_{3}\right)$, notice that $(a, b) \in E$ and $w+z p_{j}=a \frac{p_{j}}{p_{3}}+b \frac{1-p_{j}}{1-p_{3}} \in Q$. Thus $f(a, b)=X$, i.e., $f$ is surjective.

Therefore $|E| \geq|\{X \subset Q: X \sim P\}|=S_{P}(Q) \geq c n^{2}$, and additionally $\left|A+{ }_{E} B\right| \leq n$ since $A+{ }_{E} B \subseteq Q$. So we have the exact hypothesis of Lemma 2 with $C_{1}=1$ and $C_{2}=c$. Let $M=N_{1}\left(c_{3}, 2 m+1\right)$ and $N_{0}=N(1, c, M)$, where $N_{1}$ and $N$ are the respective threshold functions of Theorem D and Lemma 2. Therefore, by Lemma 2 there are arithmetic progressions $\mathcal{G}_{1}, \mathcal{G}_{2}$ of length $l \geq M$ and common difference such that

$$
\left|E \cap\left(\mathcal{G}_{1} \times \mathcal{G}_{2}\right)\right| \geq c_{3} l^{2} .
$$

Suppose $\mathcal{G}_{i}=\left\{u_{1}+j z: 0 \leq j<l\right\}$ for $i=1,2$. Identify $\mathcal{G}_{1} \times \mathcal{G}_{2}$ with $[l] \times[l]$ and $E \cap\left(\mathcal{G}_{1} \times \mathcal{G}_{2}\right)$ with the corresponding subset of $[l] \times[l]$. Now, since $l>M=N_{1}\left(c_{3}, 2 m+1\right)$ then using (6) as the hypothesis for Theorem D we conclude that $E \cap\left(\mathcal{G}_{1} \times \mathcal{G}_{2}\right)$ contains a $(2 m+1) \times(2 m+1)$ square sublattice $S_{1} \times S_{2}$. Suppose $S_{i}=\hat{u}_{i}+t z I_{m}$ for $i=1,2$, with $t$ a positive integer and $\hat{u}_{1} \in \mathcal{G}_{1}$, $\hat{u}_{2} \in \mathcal{G}_{2}$. Since $S_{1} \times S_{2} \subseteq E$ we have

$$
L_{j}:=\frac{p_{j}}{p_{3}} S_{1}+\frac{1-p_{j}}{1-p_{3}} S_{2} \subset Q \text { for } 1 \leq j \leq k .
$$

Hence $\bigcup_{j=1}^{k} L_{j}$ is the required $G_{P}(m)$ set contained in $Q$.

Remark. We actually proved that $Q$ contains a $G_{P}(m)$ set for every choice of the triplet $p_{1}, p_{2}, p_{3}$.

Acknowledgments. We are grateful to J. Beck and N. Simányi for sharing with us their knowledge on the applications of Ergodic Theory to Combinatorial Number Theory. We also thank the referees for their valuable suggestions to improve the presentation.

\section{References}

[1] B. Ábrego and S. Fernández-Merchant, On the Maximum Number of Equilateral Triangles I, Discrete and Computational Geometry 23 (2000), 129-135.

[2] A. Balog and E. Szemerédi, A Statistical Theorem of Set Addition. Combinatorica 14 (1994), 263-268. 
[3] G. Elekes and P. Erdős, Similar Configurations and Pseudogrids. In Intuitive Geometry. Colloquia Mathematica Societatis János Bolyai. North Holland, Amsterdam, (1994), 85-104.

[4] P. Erdős and G. Purdy, Some Extremal Problems in Geometry. Journal of Combinatorial Theory 10 (1971), 246-252.

[5] P. Erdős and G. Purdy, Some Extremal Problems in Geometry III. Proc. $6^{\text {th }}$ Southeastern Conference in Combinatorics, Graph Theory and Comp. (1975), 291-308.

[6] P. Erdős and G. Purdy, Some Extremal Problems in Geometry IV. Proc. $7^{\text {th }}$ Southeastern Conference in Combinatorics, Graph Theory and Comp. (1976), 307-322.

[7] G. A. Freiman, Foundations of a Structural Theory of Set Addition, Translation of Mathematical Monographs vol. 37. American Mathematical Society, Providence, R.I., USA, 1973.

[8] H. Fürstenberg and Y. Katznelson, An ergodic Szemerédi theorem for commuting transformations. Journal d'Analyse Mathématique 34 (1978), 275-291.

[9] M. Laczkovich and I.Z.Ruzsa, The Number of Homothetic Subsets, The mathematics of Paul Erdős II, Springer Verlag 1997, 294-302.

[10] M. B. Nathanson, Additive Number Theory: Inverse Problems and the Geometry of Sumsets, Springer Verlag (1996).

[11] I. Z. Ruzsa, Generalized Arithmetic Progressions and Sum Sets, Acta Mathematica Hungarica 65 (1994), 379-388.

[12] E. Szemerédi, On sets of integers containing no $k$ elements in arithmetic progression. Acta Arithmetica 27 (1975), 199-245. 